\title{
Physiological Response of Imagery Running with or without an Avatar in 3D Virtual Reality: A Preliminary Study
}

\author{
Kyung Ji Moon', Myung Chul Lee', Upyong Hong², Soo Rim Noh³, Chang Hoon Park', Kyung \\ Hun $\operatorname{Han}^{1 *}$ \\ ${ }^{1}$ Division of Sport Science, Pusan National University, Republic of Korea \\ ${ }^{2}$ Department of Media and Communication, Konkuk University, Republic of Korea \\ ${ }^{3}$ Department of Psychology, Chungnam National University, Republic of Korea \\ ${ }^{4}$ Department of Game Engineering, Hoseo University, Republic of Korea
}

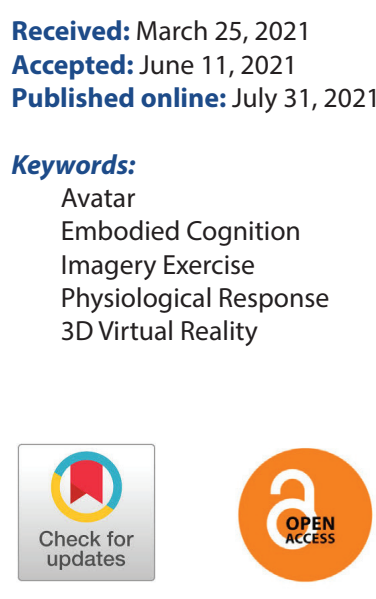

\section{ABSTRACT}

OBJECTIVES The present study is aimed at observing the physiological response imagery running with an avatar in 3D virtual reality (VR) and to explore the differences in physiological responses between imagery running with and without an avatar in 3DVR.

METHODS We randomly assigned 32 healthy adults to either a group with Avatar $(n=19)$ or a group without $(n=13)$. The group with avatar performed imagery exercise with an avatar in 3DVR and the group without Avatar performed it without an avatar in 3DVR. Both groups were instructed to mentally imagine performing running in VR without executing actual physical movements. We recorded electro-physiological data before, during, and after the 20-min intervention. We also measured the participants' level of presence and intensity of experienced cybersickness.

RESULTS In the group with Avatar, all physiological responses increased from the resting to the intervention period. The group with Avatar also showed a higher level of presence and fewer cybersickness symptoms than the group without Avatar.

CONCLUSIONS The results suggest imagery running with an avatar in 3D VR might be effective as an alternative exercise.

(c) The Asian Society of Kinesiology and the Korean Academy of Kinesiology

\section{Introduction}

Motor imagery training is the conscious mental process of a movement without any muscular execution [1]. In the field of sports, many athletes use imagery training to improve their skills, and many studies have shown such effects [2-6]. Fortes et al. [2] and Rodgers et al. [6] showed the effects of imagery

*Correspondence: Kyung-Hun Han, Division of Sport Science, Pusan National University, Busan, South Korea; Tel: +82-51-510-2721; Fax: +82-51-510-3746; E-mail: franzhan@pusan.ac.kr training on young volleyball players' passing decision-making performance and on skating performance, respectively. As imagery training is widely used as an efficient training tool for improving sports skills, one might wonder how these effects come about, considering that athletes only imagine their movements mentally without any real physical motion.

Studies in the field of neuroscience usually explain the effects of imagery training on the basis of several neurophysiological approaches [7-13]. The first is the 
psychoneuromuscular theory, which states that the muscles activated during motor execution are also activated during motor imagery [8]. The second approach is based on autonomic responses, postulating that imagery training may cause changes in autonomic activation, such as in respiration, heart rate, and skin conductance, in the similar way actual physical movements do [7]. The last approach uses the mirror neuron system to explain the effects of imagery training, suggesting that mirror neurons are activated for specific goaloriented behaviors, irrespective of whether the behaviors are produced by oneself or others [14]. Based on these three neurophysiological approaches, the effectiveness of imagery training can be neurophysiologically and physically validated.

This raises the question whether imagery training would be possible to apply for the general public as it is for athletes. To investigate this, we needed to change the concept of imagery "training" to that of imagery "exercise," because according to the mirror neuron system approach, mirror neurons react to object-oriented motor imagery and not simply to emotionally meaningful behavior $[10,14]$. In this sense, the concept of "training" which for athletes means to improve some specific performance skill, cannot inspire the same motivation in members of the general public who do not need to undergo training like athletes. In contrast, the concept of "exercise" may strongly motivate them to use motor imagery, since people usually exercise with a personal goal in mind, such as losing weight, building muscle strength, or improving life satisfaction. Therefore, the current study used the concept of imagery exercise(running) instead of imagery training to verify the possibility of application of motor imagery for the general public.

Along with different levels of motivation, it is also important to consider how vividly participants imagine physical movements. Jeannerod [15] suggested that physiological response changes could be related to the vividness of the imagined movement. However, the vividness of imagery seems to differ between elite athletes and nonelite athletes. Eton, Gilner, and Munz [16] reported that non-elite athletes showed less vivid imagery than elite athletes. This means that for the general public, it might be even more difficult to go through only conscious mental rehearsals without actual physical movements. To overcome this problem, we used a virtual reality (VR) technology setup, which can dynamically present situations that are difficult to realize experimentally. We therefore developed an imagery running with an avatar in $3 \mathrm{D} V \mathrm{VR}$, and the avatar in $3 \mathrm{D} \mathrm{VR}$ played a role as motor imagery. Based on this, the present study is aimed at observing the physiological response in experience of imagery running with an avatar in $3 \mathrm{D}$ VR.

However, to ensure the effectiveness of VR technology, it is important to explore how effectively people get involved and immersed in motor imagery in 3D VR during the imagery running. Many studies with VR have focused on perspective and on the question whether first-person perspective (1PP) or third-person perspective (3PP) is more effective [17-21]. According to Kokkinara et al. [20], when participants were seated without physical movements and watched a walking virtual body, 1PP led to a higher sense of body ownership, walking, and agency than 3PP. However, their study focused on the participant's sense of illusory agency and not on kinesiological factors. Similar to the results of Kokkinara et al's study[20], other studies have reported that 1PP led to a much higher sense of embodiment and spatial presence during VR than 3PP, with most studies focusing on the relationship between gamification and VR technology $[18,22$, 23]. Havranek et al. [22] also reported that during VR video gaming, participants showed higher ratings of spatial presence during a $1 \mathrm{PP}$ condition than during a $3 \mathrm{PP}$ condition.

In contrast to these findings, some other studies have reported that 3PP was more effective in VR than 1PP [19, 24, 25]. Galvan Debarba et al. [24] claimed that during full-body reaching tasks, 3PP can lead to a higher sense of embodiment into the virtual body. Huang et al. [19] also used a visual virtual environment for rehabilitation and found that 3PP was more effective in shifting weight. These previous studies show that 3PP can be more effective in situations where kinesiological effects are required, such as fitness, full-body reaching tasks, and rehabilitation, than in gaming situations. Particularly, in the present study, physical movements were not required to verify the effectiveness of motor imagery exercises with an avatar in $3 \mathrm{D} \mathrm{VR}$, and the participants were members of the general public, not athletes. Therefore, we hypothesized that a 
3PP task would be more effective in the present study.

In human movement, it is considered that visualizing the motor imagery for the specific motion is important. This is because the motor system has the ability to copy and reproduce another's motion [26]. Therefore, when people experience movements from a 3PP, they are able to recognize them more accurately than when they experience movements from a 1PP [27]. Huang et al. [19] suggested that 3PP is more useful in rehabilitation than 1PP. Furthermore, in VR, 3PP leads to less simulator sickness than 1PP [28]. Therefore, in previous studies on the effect of different perspectives in VR, findings might have depended on the specific research purpose. In the present study, we additionally compared $3 \mathrm{D}$ VR imagery running with an avatar with $3 \mathrm{D}$ VR imagery running without an avatar to verify which perspective was more effective.

In sum, our main aim was to observe the physiological response of imagery running with an avatar in $3 \mathrm{D}$ VR. The second goal of this study was to examine the differences in physiological responses between imagery running with and without an avatar in 3D VR. Additionally, we also assessed differences in the level of perceived presence between imagery running with and without an avatar in $3 \mathrm{D}$ VR.

\section{Methods}

\section{Participants}

This study recruited neurologically and physically healthy men and women aged between 19 and 35 years. Thirty-two participants were randomly assigned into either the group with Avatar ( $\mathrm{n}=19$; mean age: $22.57 \pm 2.26$ years, nine men, 10 women), who performed the imagery running with an avatar in $3 \mathrm{D}$ VR, or the group without Avatar $(n=13$; mean age: $21.38 \pm 1.75$ years, five men, eight women), who performed the imagery running without an avatar in $3 \mathrm{D}$ VR. A total of 39 people were recruited at the beginning of experiment, with 20 assigned to the experimental group and 19 to the control group. Among them, one experimental group participant and three control group participants fell asleep during the experiment. Two additional control participants were eliminated due to motion sickness. In addition, data from one control participant was not collected due to technical problems, leaving 13 control group participants and 19 experimental group participants in the data analysis. The study was approved by Institutional Review Board (IRBPNU IRB/2018_84_HR) and written informed consent for study participation was obtained from all participants.

\section{Materials and Apparatus}

The 3D VR programs for both the with and without avatar groups used an HTC Vive VR (HTC Corporation, New Taipei City, Taiwan), which was composed of one head mounted display (HMD), a pair of hand controllers, and headphones, and the SteamVR Unity Plugin. The HMD in this study had a display resolution of $1080 \times 1200$.

The electro-physiological signal data was obtained by ProComp Infiniti (Thought Technology Ltd., Montreal, Canada) and the Biograph Infiniti Software system (version 6.5.0). Regarding the EMG sensor, The MyoScan-Pro sensor was used. Its active range is from 20 to $500 \mathrm{~Hz}$. It can record SEMG(surface electromyography) signals of up to 1600 microvolts $(\mu \mathrm{V})$, RMS. On the sensor, there is a small switch with three positions: $400 \mathrm{~N}, 1600 \mathrm{~W}$ and $400 \mathrm{~W}$. At the $400 \mathrm{~W}$ or $400 \mathrm{~N}$ positions, the sensor's amplitude scale is $400 \mu \mathrm{V}$, which allows for a good resolution for most of the body's muscles. The larger body muscles can produce microvolt readings of up-to $1600 \mu \mathrm{V}$. The noise caused by heartbeat recording upper body muscles can be filtered out by moving the switch to the $400 \mathrm{~N}$ (narrow bandwidth) position. In this position, the sensor will only be sensitive to frequencies between 100 and $200 \mathrm{~Hz}$.

\section{Measurements}

To verify physiological responses, we obtained electro-physiological signal data from six channels: electrocardiography (ECG); electromyography (EMG) of the left side of the biceps brachii, rectus femoris, and lateral gastrocnemius; blood volume pulse (BVP); and skin conductance (SC).

For the immersion and stability of both 3D VR programs used in this study, we administered the Presence Questionnaire (PQ) [29] and the Simulator Sickness Questionnaire (SSQ) 
[30], which were modified and translated to Korean.

\section{Intervention}

Both groups were instructed to mentally imagine that they were exercising in VR without executing any actual physical movements. The 3D VR programs used for both the two groups were implemented for 20 minutes.

As depicted in Figure 1A, the 3D VR program for the group with Avatar was an avatar jogging simulation. In this program, participants could select an avatar of the same sex and control the speed of the avatar with controllers. They were also able to hear the footsteps at the controlled speed of the avatar. Furthermore, before the intervention, the participants were instructed to focus on the avatar's leg movements. The $3 \mathrm{D} V R$ program for the group without Avatar was a jogging simulation with only tracks but no avatar, as shown in $<$ Figure $1>$. The group without Avatar did thus not select an avatar, control the speed of an avatar, hear the footsteps, and receive any further instructions.

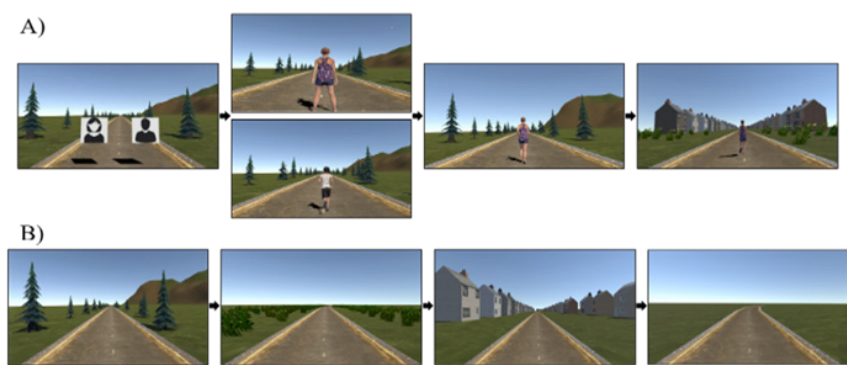

Figure 1. The 3D VR running simulation. A) Running simulation for the group with Avatar. B) Running simulation for the group without Avatar.

\section{Procedure}

This study was performed as a single-blind, randomized controlled trial. Participants were recruited through advertisements on the PNU internet website. All participants took the first screening test by phone. After the first screening, participants who passed the inclusion criteria were asked to visit the laboratory. A total of thirty-two participants were randomly assigned to either the group with Avatar or the group without Avatar.

At the site visit for the experiment, the participants took the screening test again, and then they received general and detailed information for the study and completed the informed consent form. Before starting the experiment, they experienced the 3D VR program used in this study to verify symptoms of cybersickness for 5 minutes.

If participants had no problem with cybersickness symptoms, they took a rest for 15 minutes and then they attached electro-physiological sensors. For measuring the electro-physiological data, all participants went through an adaptation period ( $5 \mathrm{~min}$ ), a pre-resting period before the intervention (5 min), the intervention period (20 min), and a post-resting period after the intervention ( $3 \mathrm{~min}$ ). After this process, the PQ and SSQ were measured to verify the immersion and stability of the 3D VR programs.

\section{Statistical analysis}

All statistical procedures were conducted using IBM SPSS 22.0 for Windows (SPSS Inc., Chicago, IL, USA). All electrophysiological data were analyzed using repeated-measures analysis of variance (RM-ANOVA) with the factors time (three levels: pre-resting, intervention, post-resting) and group (two levels: with, without avatar). To compare mean differences in the PQ and SSQ scores between the two groups, independent $t$-tests were used.

\section{Results}

Results of physiological responses are summarized in $<$ Table 1>. For all electro-physiological data, the group with Avatar showed higher electro-physiological responses during the intervention (imagery running with an avatar in 3D VR) than during the pre- and post-resting periods before and after the intervention. Moreover, ECG and BVP findings were significantly different between the avatar with and without. The EMG findings showed medium effect sizes (partial $\eta 2>$ .06) for a time-by-group interaction, although there were no statistically significant differences between the groups.

Results from the PQ and SSQ are summarized in <Table 2> In terms of the $\mathrm{PQ}$, the group with Avatar $(M=160.21$, $S D=22.55)$ showed higher levels of presence than the group without Avatar $(M=147.23, S D=11.09)$, although there was no significant difference between the two groups $(t(30)=1.91$, $p>.05)$. In terms of the SSQ, the group with Avatar showed 
Table 1. Changes in electro-physiological responses

\begin{tabular}{|c|c|c|c|c|c|c|c|c|}
\hline \multirow{2}{*}{ Variables } & \multicolumn{3}{|l|}{ with Avatar } & \multicolumn{3}{|c|}{ without Avatar } & \multicolumn{2}{|c|}{ RM-ANOVA } \\
\hline & $\begin{array}{l}\text { Pre-resting } \\
M(S D)\end{array}$ & $\begin{array}{l}\text { Intervention } \\
M(S D)\end{array}$ & $\begin{array}{l}\text { Post-resting } \\
M(S D)\end{array}$ & $\begin{array}{l}\text { Pre-resting } \\
M(S D)\end{array}$ & $\begin{array}{l}\text { Intervention } \\
M(S D)\end{array}$ & $\begin{array}{l}\text { Post-resting } \\
M(S D)\end{array}$ & $\begin{array}{l}\text { Time } \\
F(p n 2)\end{array}$ & $\begin{array}{l}T \times G \\
F(p \eta 2)\end{array}$ \\
\hline $\begin{array}{l}\text { ECG } \\
\text { (beats/min) }\end{array}$ & $74.37(7.42)$ & $81.24(9.17)$ & $75.91(8.64)$ & $72.86(6.15)$ & $75.86(6.23)$ & $75.22(7.95)$ & $\begin{array}{l}24.09^{* * *} \\
(.445)\end{array}$ & $\begin{array}{l}6.11^{* *} \\
(.169)\end{array}$ \\
\hline EMG A $(\mu \mathrm{V})$ & $0.69(1.12)$ & $\begin{array}{l}1.37 \\
(1.52)\end{array}$ & 0.78 (1.19) & $0.31(0.11)$ & $\begin{array}{l}0.44 \\
(0.26)\end{array}$ & $0.37(0.14)$ & $\begin{array}{l}4.57^{*} \\
(.132)\end{array}$ & $\begin{array}{l}2.28 \\
(.071)\end{array}$ \\
\hline EMG B $(\mu \mathrm{V})$ & $3.20(10.35)$ & $5.89(15.67)$ & $6.00(16.71)$ & $0.47(0.93)$ & $\begin{array}{l}0.62 \\
(0.92)\end{array}$ & $0.35(0.60)$ & $\begin{array}{l}2.17 \\
(.067)\end{array}$ & $\begin{array}{l}2.16 \\
(.067)\end{array}$ \\
\hline EMG C $(\mu \mathrm{V})$ & $1.09(1.26)$ & $\begin{array}{l}1.50 \\
(1.53)\end{array}$ & $1.01(1.12)$ & $0.78(0.26)$ & $\begin{array}{l}0.73 \\
(0.15)\end{array}$ & $0.70(0.13)$ & $\begin{array}{l}2.41 \\
(.074)\end{array}$ & $\begin{array}{l}2.36 \\
(.073)\end{array}$ \\
\hline $\begin{array}{l}\text { BVP } \\
\text { (pulse/min) }\end{array}$ & 74.28 (7.29) & $81.13(9.46)$ & $75.45(8.32)$ & 72.69 (6.29) & $75.76(6.24)$ & $74.95(7.68)$ & $\begin{array}{l}24.13^{* * *} \\
(.446)\end{array}$ & $\begin{array}{l}6.23^{* *} \\
(.172)\end{array}$ \\
\hline $\mathrm{SC}(\mu \mathrm{s})$ & $1.47(1.31)$ & $\begin{array}{l}2.75 \\
(2.32)\end{array}$ & $3.28(2.63)$ & $0.50(0.22)$ & $\begin{array}{l}1.00 \\
(0.74)\end{array}$ & $2.42(2.54)$ & $\begin{array}{l}19.35^{* * *} \\
(.392)\end{array}$ & $\begin{array}{l}1.31 \\
(.042)\end{array}$ \\
\hline
\end{tabular}

Note. ${ }^{*} p<.05,{ }^{* *} p<.01,{ }^{* * *} p<.001 ; M=$ Mean; $S D=$ Standard Deviation; $F=F$-value; $\mathrm{T} \times \mathrm{G}=$ time $\times$ group interaction; $p$ 2: partial eta squared; ECG: electrocardiography; EMG: electromyography, A: the left biceps brachii, B: the left rectus femoris, C: the left lateral gastrocnemius; BVP: blood volume pulse; SC: skin conductance.

Table 2. Differences in PQ and SSQ scores between the avatar with and without

\begin{tabular}{lllll}
\hline Variables & $\begin{array}{l}\text { with Avatar } \\
\boldsymbol{M}(\boldsymbol{S D})\end{array}$ & $\begin{array}{l}\text { without Avatar } \\
\boldsymbol{M}(\boldsymbol{S D})\end{array}$ & $\boldsymbol{t}$-value & $\boldsymbol{p}$-value \\
\hline $\mathrm{PQ}$ & $160.21(22.55)$ & $147.23(11.09)$ & 1.91 & .065 \\
$\mathrm{SSQ}$ & $4.68(4.01)$ & $5.23(8.24)$ & -.25 & .804 \\
\hline
\end{tabular}

Note. $M=$ Mean; $S D=$ Standard Deviation; $P Q$, Presence Questionnaire; SSQ, Simulator Sickness. Questionnaire

fewer symptoms of cybersickness $(M=4.68, S D=4.01)$ than the group without Avatar $(M=5.23, S D=8.24)$, but there was no statistically significant difference between the two groups $(t(30)=-.25, p>.05)$; both groups showed few levels of cybersickness symptoms overall.

\section{Discussion}

This study is aimed at observing the physiological response of imagery running with an avatar in $3 \mathrm{D}$ VR and to explore the differences between 1PP and 3PP conditions in terms of changes in physiological responses. The results of this study showed that (I) the imagery running with an avatar in $3 \mathrm{D} V \mathrm{VR}$ led to increased physiological response change patterns from the resting period to the intervention, similar to that seen in physical exercise; (II) the group with Avatar showed larger changes in physiological responses than the group without Avatar; and (III) the group with Avatar showed higher levels of presence in VR than the group without Avatar.

First, all physiological responses showed increases from the resting period to the intervention period in the group with Avatar, in which the participants performed the imagery running with an avatar in $3 \mathrm{D}$ VR. These results are in line with those of previous studies demonstrating the physiological effectiveness of motor imagery [31-33]. Papadelis et al. [33] reported that motor imagery training increased participants' heart rate and electromyographic activity. Furthermore, according to the psychoneuromuscular theory, muscles can be activated during motor imagery training without real physical movements [8]. Based on this theory, Guillot et al. [32] also reported the effectiveness of motor imagery without any actual movements on muscle activation. Considering the findings of previous studies, our imagery running with an avatar in $3 \mathrm{D}$ VR led to physiological effects similar to those reported for motor imagery training.

In addition, these increased physiological response change patterns are similar to the physiological response change patterns obtained with real physical exercise. When people exercise, they show increases in heart rate, muscle activity, and activation of the sympathetic nervous system compared to when they take a rest, and these increases decrease again 
during a rest period after the exercise. Our data show a similar physiological pattern, in that all physiological responses increased from the resting period before the intervention to the intervention period and in that the increases in ECG, biceps brachii, lateral gastrocnemius, and BVP values decreased again from the intervention period to the following resting period. Many previous studies have reported increases in physiological responses during physical activities or exercise periods compared to those at rest [34-39]. Serrador et al. [37] reported that mean heart rates and EMG activation were greater during a physically active state than during rest. Vargas et al. [39] also found increases in response changes from the period before the exercise to the exercise period in terms of skin wettedness. The results of the current study are thus partially in line with those of previous studies, even though the present study required participants to only mentally exercise using motor imagery without any real physical movements. This indicates that imagery running with an avatar in $3 \mathrm{D}$ VR leads to physiological response change patterns in the similar way real physical running does, which means that it can be applied as a new type of exercise.

Secondly, to observe the physiological response of being embodied into an avatar in 3D VR (3PP), we compared imagery running with an avatar in $3 \mathrm{D} V \mathrm{VR}$ to imagery running without an avatar in 3D VR (1PP). The group with Avatar that performed the imagery running with an avatar in $3 \mathrm{D}$ VR showed greater increases in ECG and BVP responses from the resting period to the intervention period than the group without Avatar that performed the imagery running without an avatar in 3D VR. Moreover, although there were no significant differences between the two groups in the present study, the effect sizes of all EMG values in the time-by-group interaction were greater than the medium size, and the EMG values in the group with Avatar showed greater increases in responses from the resting period to the intervention period than those in the group without Avatar. These results indicate that $3 \mathrm{PP}$ is more effective than $1 \mathrm{PP}$ in inducing physiological response changes similar to those observed with actual exercise. Jeannerod [15] stated that the vividness of imagery is important to induce physiological responses. Vergeer and Roberts [40] also suggested that movement imagery could be effective for improving physiological effects by increasing the vividness of the imagery. In agreement with previous studies, the present study also shows that imagery running from a $3 \mathrm{PP}$ induced greater physiological response changes than imagery running from a $1 \mathrm{PP}$. This suggests that in the absence of physical movements, presenting motor imagery with an avatar in 3D VR improved the vividness of the motor imagery. This presumably made it easier for the group with Avatar to understand the motion, to feel a sense of embodiment, and to only mentally execute the exercise without actual movements.

In the PQ assessment, the group with Avatar showed a higher level of presence in VR than the group without Avatar, although there was no significant difference between the two groups. This is inconsistent with the findings of previous studies that showed higher effectiveness for the 1PP than for the 3PP $[18,20,22,23]$. The fact that some previous studies were related to gaming might explain some of the differences between the present findings and previous findings. In the present study, it was important for participants to feel embodied into the motor imagery to understand and learn the kinesiological movements. This is why the vividness of the imagery was relevant and why the imagery running with an avatar in 3D VR was more effective than that without an avatar in $3 \mathrm{D}$ VR. This finding is therefore partially in line with those of previous studies on kinesiological effects that showed higher effectiveness of the 3PP than the 1PP $[19,24,25]$. Especially, according to Kokkinara et al. [20], although the authors showed the effectiveness of the 1PP in illusions of body ownership and agency compared with the 3PP, their participants initially saw the avatar's whole body through a mirror in a virtual environment, watching the avatar walking from a $1 \mathrm{PP}$. This suggests that there were effects of the 1PP on the participants' experience of illusory agency since participants firstly understood the whole virtual body by showing the virtual body through the mirror in the VR before performing the program from a $1 \mathrm{PP}$. Therefore, this finding indicates that presenting motor imagery from a $3 \mathrm{PP}$ can be more effective for the participant's sense of embodiment and presence when there are no actual physical movements. However, we found no significant difference between the two groups in the PQ score, which is probably due to our small 
sample size. Therefore, future studies with larger sample sizes are necessary to further investigate this issue.

The SSQ assessment showed no significant difference between the two groups. Both groups showed few levels cybersickness symptom, which means that both 3D VR programs used in this study are safe to use and do not induce cybersickness.

In conclusion, this study demonstrates the physiological response of imagery running with an avatar in $3 \mathrm{D} V \mathrm{VR}$ and shows significant differences between the 1PP and 3PP. Furthermore, we demonstrate the effectiveness of imagery running with an avatar in $3 \mathrm{D}$ VR in terms of the level of presence in VR compared to that of imagery without an avatar in 3D VR. This is the first study to address the usability of a combination of motor imagery and 3D VR technology in members of the general public as an alternative exercise. Contrary to previous studies on imagery training, this study complemented imagery training with VR technology and observed its physiological response while also comparing 1PP and 3PP. Furthermore, this is also the first study to adapt the concept of imagery exercise used in athletes to one of imagery training to make the motor imagery approach useful for the general public.

Notwithstanding the intriguing results of the current study, it has some limitations. Since this study had a small sample size, which makes it difficult to generalize its findings, further research with larger sample sizes is needed to validate the study findings. In Addition, the difference in physiological response by sex was not observed because the sex ratio was not matched and the physiological characteristics of subjects were not collected. This study observed the physiological response of only one 20-minute experience, then further studies are needed to verify the long-term effectiveness of imagery running with an avatar in $3 \mathrm{D}$ VR in terms of physiological responses.

\section{Acknowledgments}

This work was supported by the National Research Foundation of Korea (NRF) grant funded by the Korea government(MSIT) (No. 2017R1C1B5018351) and by the Ministry of Education of the Republic of the Korea and National Research Foundation of Korea(NRF2018S1A5B6069474).

\section{References}

1. Grabherr L, Jola C, Berra G, Theiler R, Mast FW. Motor imagery training improves precision of an upper limb movement in patients with hemiparesis. NeuroRehabilitation. 2015; 36(2):157-166.

2. Fortes LS, Freitas-Júnior CG, Paes PP, et al. Effect of an eight-week imagery training programme on passing decision-making of young volleyball players. Int J Sport Exerc Psychol. 2018; 18(9):120-128.

3. Fortes LD, Almeida SS, Nascimento Junior JRAD, Vieira LF, Lima-Júnior D, Ferreira MEC. Effect of motor imagery training on tennis service performance in young tennis athletes. Rev Psicol Deporte. 2019; 28(1):157-168.

4. Lee CY, Lee SK, Song JH. The effects of imagery training on shooting and penalty shot of ice hockey players. KJSS. 2008; 19(4):204-213.

5. Robin N, Dominique L, Toussaint L, Blandin Y, Guillot A, Her ML. Effects of motor imagery training on service return accuracy in tennis: The role of imagery ability. International Journal of Sport and Exercise Psychology. 2007; 5(2):175-186.

6. Rodgers W, Hall C, Buckolz E. The effect of an imagery program on imagery ability, imagery use, and figure skating performance. J Appl Sport Psychol. 1991; 3(2):109-125.

7. Decety J. The neurophysiological basis of motor imagery. Behav Brain Res. 1996; 77:45-52.

8. Driskell JE, Copper C, Moran A. Does mental practice enhance performance? J Appl Psychol. 1994; 79(4):481492.

9. Gerardin E, Sirigu A, Lehéricy S, et al. Partially overlapping neural networks for real and imagined hand movements. Cereb Cortex. 2000; 10(11):1093-1104.

10. Li L, Wang J, Xu G, Li M, Xie J. The study of objectoriented motor imagery based on EEG suppression. PLoS One. 2015; 10(12):e014456. 
11. Mulder T, Zijlstra S, Zijlstra W, Hochstenbach J. The role of motor imagery in learning a totally novel movement. Exp Brain Res. 2004; 154:211-217.

12. Munzert J, Lorey B, Zentgraf K. Cognitive motor processes: The role of motor imagery in the study of motor representations. Brain Res Rev. 2009; 60(2):306-326.

13. Silva S, Borges L, Santiago L, Lucena L, Lindquist AR, Ribeiro T. Motor imagery for gait rehabilitation after stroke. Cochrane Database Syst Rev. 2018; 2015(5):CD013019.

14. Aziz-Zadeh L, Ivry RB. The human mirror neuron system and embodied representations. Adv Exp Med Biol. 2009; 629:355-376.

15. Jeannerod M. The representing brain: Neural correlates of motor intention and imagery. Behav Brain Sci, 1994; 17(2):187-245.

16. Ethon DT, Gilner FH, Munz DC. The measurement of imagery vividness: A test of the reliability and validity of the vividness of visual imagery questionnaire. Journal of Mental Imagery, 1998; 22(3-4):125-136.

17. Falconer CJ, Slater M, Rovira A, et al. Embodying compassion: A virtual reality paradigm for overcoming excessive self-criticism. PLoS One. 2014; 9(11):e111933.

18. Gorisse G, Christmann O, Amato EA, Richir S. Firstand third-person perspectives in immersive virtual environments: Presence and performance analysis of embodied users.Front Robot AI. 2017; 4:33.

19. Huang CH, Pei C, Sun TL. Influence of the perspectives on the movement of one-leg lifting in an interactivevisual virtual environment: A pilot study. PLoS One. 2016; 11(9):e0163247.

20. Kokkinara E, Kilteni K, Blom KJ, Slater M. First person perspective of seated participants over a walking virtual body leads to illusory agency over the walking. Sci Rep. 2016; 6(1):1-11.

21. Salamin P, Tdi T, Blanke O, Vexo F, Thalmann D. Quantifying effects of exposure to the third and firstperson perspectives in virtual-reality-based training. IEEE Transactions on Learning Technologies. 2010; 3(3):272-276.

22. Havranek M, Langer N, Cheetham M, Jäncke L. Perspective and agency during video gaming influences spatial presence experience and brain activation patterns. Behav Brain Funct. 2012; 8(1):34.

23. Ishiguro T, Suzuki C, Nakakoji H, Funagira Y, Takao M. Immersive experience influences eye blink rate during virtual reality gaming. Pol Psychol Bull. 2019; 50(1):4953.

24. Galvan Debarba H, Molla E, Herbelin B, Boulic R. Characterizing embodied interaction in First and Third Person perspective viewpoints. Proceedings of 2015 IEEE Symposium on 3D User Interfaces (3DUI). 2015; $67-72$.

25. Lugrin JL, Landeck M, Latoschik ME. Avatar embodiment realism and virtual fitness training. Proceedings of 2015 IEEE Virtual Reality (VR). 2015; 225-226.

26. Shiffrar M, Heinen T. Athletic ability changes action perception: Embodiment in the visual perception of human movement. German Journal of Sport Psychology. 2010; 17(4):130-142.

27. Prasad S, Shiffrar M. Viewpoint and the recognition of people from their movements. J Exp Psychol Human, 2009; 35(1):39-49.

28. Monteiro D, Liang HN, Xu W, Brucker M, Nanjappan V, Yue Y. Evaluating enjoyment, presence, and emulator sickness in VR games based on first-and third-person viewing perspectives. Comput Animat Virt W. 2018; 29(3-4):e1830.

29. Witmer BG, Singer MJ. Measuring presence in virtual environments: A presence questionnaire. Presence, 1998; 7(3):225-240.

30. Kennedy RS, Lane NE, Berbaum KS, Lilienthal MG. Simulator sickness questionnaire: An enhanced method for quantifying simulator sickness. Int J Aviat Psychol. 1993; 3(3):203-220.

31. Bakker FC, Boschker MS, Chung T. Changes in muscular activity while imagining weight lifting using stimulus or response propositions. J Sport Exerc Psychol. 1998; 20(3):313-324.

32. Guillot A, Lebon F, Rouffet D, Champely S, Doyon J, Collet C. Muscular responses during motor imagery as a function of muscle contraction types. Int J 
Psychophysiol. 2007; 66:18-27.

33. Papadelis C, Kourtidou-Papadeli C, Bamidis P, Albani M.

Effects of imagery training on cognitive performance and use of physiological measures as an assessment tool of mental effort. Brain Cognition, 2007; 64(1):74-85.

34. Choi BS, Han SW. Effects of resting periods between exercise sets during isokinetic exercise on muscle performances and physiological variables in middleaged women. Phys Ther Korea. 2001; 8(3):77-95.

35. Katayama K, Goto K, Ishida K, Ogita F. Substrate utilization during exercise and recovery at moderate altitude. Metabolism. 2010; 59(7):959-966.

36. Michelsen JS, Lund MC, Alkjær T, Finni T, Nielsen JB, Lorentzen J. Wearable electromyography recordings during daily life activities in children with cerebral palsy. Dev Med Child Neurol. 2020; 62(2):714-722..
37. Serrador JM, Finlayson HC, Hughson RL. Physical activity is a major contributor to the ultra low frequency components of heart rate variability. Heart, 1999; 82(6):e9.

38. Ulucan S, Kaya Z, Keser A, Katlandur H, Karanfil M, Ateş $\dot{I}$. Deterioration of heart rate recovery index in patients with erectile dysfunction. Anatol J Cardiol. 2016; 16(4):264-269.

39. Vargas NT, Chapman CL, Johnson BD, Gathercole $\mathrm{R}$, Schlader ZJ. Skin wettedness is an important contributor to thermal behavior during exercise and recovery. Am J Physiol Regul Integr Comp Physiol. 2018; 315(5):925-933.

40. Vergeer I, Roberts J. Movement and stretching imagery during flexibility training. J Sport Sci. 2006; 24(2):197208. 